\title{
Dynamic Balance in Athletes With Intellectual Disability: Effect of Dynamic Stretching and Plyometric Warm-Ups
}

\author{
Ghada Jouira, Selim Srihi, Fatma Ben Waer, Haithem Rebai, and Sonia Sahli
}

\begin{abstract}
Context: Athletes with intellectual disability (ID) have a high risk of injury while participating in various sports. Warm-up (WU) is the most preventive measure to reduce injuries in sports. Objective: To investigate the effects of dynamic stretching WU (DS-WU) and plyometric WU (PL-WU) on dynamic balance in athletes with ID. Design: Crossover study. Setting: Research laboratory. Participants: A total of 12 athletes with ID (age 24.5 [3.22] y, height 165.7 [8.4] cm, weight 61.5 [7.1] kg, intelligence quotient 61.1 [3.5]). Main Outcome Measures: Dynamic balance was assessed using the Star Excursion Balance Test (SEBT) at pre-WU, post-WU, and 15 minutes post-WU for both the DS-WU and the PL-WU. A 2-way analysis of variance ( 3 sessions $\times 2$ WU methods) with repeated-measures was used in this study. Results: Following the DS-WU, participants demonstrated significant improvements in the SEBT composite score post-WU $(89.12 \%$ [5.54\%] vs $87.04 \%$ [5.35\%]; $P<.01)$ and at 15 minutes post-WU $(89.55 \%$ [5.28\%] vs $87.04 \%, P<.01)$ compared with pre-WU. However, no significant difference between these two post-WU scores (post-WU and 15 min post-WU) was found. For the PL-WU, participants demonstrated a significant decrease in the SEBT composite score at post-WU $(85.95 \%$ [5.49\%] vs $87.02 \%$ [5.73\%]; $P<.05$ ); however, these scores increased significantly at 15 minutes post-WU $(88.60 \%$ [5.42\%] vs 87.02\% [5.49\%]; $P<.05)$ compared with that at pre-WU. The SEBT composite scores are significantly higher in the DS-WU than in the PL-WU at both post-WU sessions $(P<.05)$. Conclusion: Both DS-WU and PL-WU could improve dynamic balance and may be recommended as WUs in athletes with ID; however, particular caution should be exercised immediately after the PL-WU.
\end{abstract}

Keywords: Star Excursion Balance Test, Paralympic, sport injuries prevention

Recently, public health began to consider the health needs of people with intellectual disability (ID). ${ }^{1}$ As a result, there has been an improvement in living conditions for these people, including a regular routine of sports practice. Today, the number of athletes with ID is increasing steadily around the world. It is noted that participating in organized sports has been associated with physical, psychological, and social benefits in people with ID. The physical benefits include improvement in muscle strength, aerobic capacity, coordination, agility, and balance. ${ }^{2}$ Participation in sport is, however, associated with a significant risk for sustaining injuries. A previous study showed that injury rates in Paralympic sports are generally high with a trend toward more injuries compared with the sport for athletes with typical development. ${ }^{3}$ Athletes with ID have cognitive impairment which reduces their ability to think rationally and understand safety concepts, such as appreciating danger or taking evasive action, which increases their risk of injuries. ${ }^{4}$ Besides, they appear to have impaired postural stability compared with the general population. ${ }^{5}$ This postural deficit has been explained through previous studies based on the deficits in visual, ${ }^{6}$ proprioceptive, ${ }^{7}$ and vestibular $^{8}$ inputs. In addition, the low balance ability has been associated with an increased risk of injuries in athletes with ID. ${ }^{9}$

Postural balance consists of 2 components, static and dynamic. While static balance manages the support base with the minimum movement, dynamic balance consists of the ability to maintain the stability of the body while performing a movement. ${ }^{10}$ It is not only required for daily life activities such as walking, stair climbing, bathing but it is also an important factor associated with athletic

The authors are with the Research Laboratory Education, Motricité, Sport et Santé (EM2S) LR19JS01, High Institute of Sport and Physical Education of Sfax, University of Sfax, Sfax, Tunisia. Jouira (jouiraghada0825@gmail.com) is corresponding author. performance and lower-extremity injury, such as sprains and muscle, tendon, and ligament strains. ${ }^{11}$ Therefore, improving balance in athletes with ID could be an important option for preventing injuries.

The most common preventive process to reduce the incidence and severity of muscle and tendon injuries in sports is performing warm-up (WU). Stretching exercises have been widely used as a training or competition WU routine to prepare the musculoskeletal system before any sports performance. In athletes with typical development, it has been shown that static stretching may have a negative effect on a variety of performance variables such as sprinting, ${ }^{12}$ agility, ${ }^{13}$ and postural balance. ${ }^{14}$

Recent studies have been focused on dynamic stretching (DS) and plyometric (PL) exercise as important modified WU methods for athletes with typical development. It has been reported that DS prepares the body for physical activity by increasing joint range of motion and blood and oxygen flow to soft tissues, thereby preventing the risk of injury during the subsequent activity. ${ }^{15}$ In addition, it challenges the balance and coordination skills that could help performance, and activates muscles during training, which subsequently improves muscle performance. ${ }^{16}$ In this context, it has been found that a stronger muscle around the joint can provide better balance and perform more efficient and explosive movements for a longer period. ${ }^{17}$

PL is a form of exercise that involves jumping, hopping, bounding, and/or skipping. It uses high-speed eccentric exercises to advance concentric muscle action, reflexive responses, and functional exercise patterns. ${ }^{18}$ According to previous studies, PL exercises can enhance muscular performance, ${ }^{19}$ flexibility, and running velocity. ${ }^{20} \mathrm{PL}$ exercises offer gain not only in strength but also in dynamic balance among athletes with typical development by challenging the neuromuscular system..$^{21,22}$ 
The positive effects of DS and PL exercises on balance in athletes with typical development have been proved previously. However, no data about these effects on athletes with ID are available, although they are more susceptible to injuries, compared with the general population, ${ }^{5}$ because of their poor balance. An understanding of these effects can provide a better understanding of the best WU method for athletes with ID in order to improve their balance and prevent them from injuries.

Hence, this study aims to investigate the effects of 2 different WU methods, DS and PL exercises, on dynamic balance in athletes with ID. We hypothesized that both WU methods would improve dynamic balance in athletes with ID.

\section{Methods}

\section{Participants}

A total of 12 athletes with mild ID (intelligence quotient between 50 and 70 according to the Wechsler Adult Intelligence ScaleFourth Edition test ${ }^{23}$ ) were recruited from an educational center, the Association of Tunisian Parents and Mentally Handicapped Friends, to participate in our study. The demographic and anthropometric characteristics of these athletes (age 24.5 [3.22] y, height $165.7[8.4] \mathrm{cm}$, weight $61.5[7.1] \mathrm{kg}$, intelligence quotient 61.1 [3.5]) were collected from their medical files, determined by the "Association of Tunisian Parents and Mentally Handicapped Friends" psychologist and by interviewing their trainer. The inclusion criteria were a middle socioeconomic status, a 4-6 years of athletic running experience (4 sessions per week with 2-3 h per session), and a high physical activity level (based on scores in the International Physical Activity Questionnaire). Athletes with any history of lower limb and/or low back injuries, neuromuscular disorders, musculoskeletal problems, and/or multiple disabilities were excluded from this study. Our recruitment strategy consisted of a 3-stage screening process. In the first stage, 20 athletes with ID were screened. In the second stage, 15 of those athletes who met the selected inclusion and exclusion criteria were selected. In the third stage, 3 of the 15 selected athletes were excluded from this study because they did not participate in the familiarization session. Eventually, 12 athletes with ID participated in our study.

The experimental protocol was explained to all participants, their parents/guardians, and the director of the educational center. A signed consent form was obtained from the participants themselves or their parents/guardians. The present study was conducted according to the Declaration of Helsinki and the protocol was fully approved by the local Ethics Committee (CPP: 0228/2020).

\section{Study Design}

Two different WU protocols were used in this study for set times over a 2-day period: DS WU (DS-WU) and PL WU (PL-WU). First, the anthropometric measurements of the athletes were collected before the WU. The test was carried out at the same hours of the day (13:30-16:00 h), which was also the habitual hour of participants' training sessions. The study design consisted of a preWU session where the dynamic balance of each participant was assessed using the Star Excursion Balance Test (SEBT); subsequently, a WU session was performed which consisted of 5 minutes of jogging and DS or PL exercises; immediately (post-WU) and 15 minutes (15 min post-WU) after the WU session, dynamic balance was assessed again. Each participant underwent $2 \mathrm{WU}$ protocols (DS-WU and PL-WU) separated by at least 48 hours to eliminate the effects of the previous WU session. Following an explanation of all the experimental procedures as well as their risks and benefits, all athletes were helpful and motivated to participate in the protocol. To further understand the testing procedure, all participants viewed an instructive video. Moreover, they made a good effort to attend WU sessions and to perform the SEBT. Before the commencement of the first testing session, all participants were familiar with the SEBT, which was necessary to reduce the change in the learning effect occurring during the test.

\section{Dynamic Balance Assessment}

To evaluate the dynamic balance of our participants, the SEBT was used. The SEBT consisted of a grid of 8 directions suspended at $45^{\circ}$ from each other. These directions are anterior (ANT), anterolateral (ALAT), lateral (LAT), posterolateral (PLAT), posterior (POST), posteromedial (PMED), medial (MED), and anteromedial (AMED). While maintaining the position of the dominant leg (the preferred kicking leg) in the center of the grid, each participant was requested to reach as far as he could in each direction by the nondominant leg, slightly touch the ground, and bring it back to the center. ${ }^{24}$ Participants performed 3 trials in each direction to minimize the learning effect, with a 2 -minute rest between trials. The best trial was considered for analysis. The trial was rebuffed and returned if participants removed their dominant foot from the center of the grid or used the reaching leg for support. To normalize the data, each reach distance was divided by the length (anterior superior iliac spine to medial malleolus) of the leg ([reach distance/length of the leg] $\times 100$ ). The SEBT composite score was calculated by dividing the sum of the normalized scores for each direction by the number of directions ([sum] all of normalized scores/8).

\section{Interventions}

DS-WU Protocol. The DS-WU protocol consisted of 5 minutes of jogging and a sequence of DS exercises commonly used in physical education and sport in athletes with typical development. ${ }^{25,26}$ Each of these exercises was adapted to our population. The exercises were simplified and adapted to our participants by reducing the complexity of the movements and performing the limb stretching exercise one by one as well by reducing the number of times these are to be performed. The participant performed each exercise 8 times for each single leg, for 3 repetitions with 10-second muscle release between each repetition. The DS exercises were:

1. Leg swing: Swing the leg forward and backward. Gradually increase the range of motion until the leg swings as high as it will comfortably go; and then repeat on the opposite side.

2. Lunge with hamstring stretch: Step with the right foot into a forward lunge. Place the hands on the floor by the front foot, then push the hips up to the ceiling and straighten the back leg to stretch. Bend the knees and return to stand, and repeat on the opposite side.

3. Hip-extensor stretch: In the standing position, move the right leg straight while the opposite arm is alternatively moved backward; and then change sides.

4. Leg flexor stretch: Stand tall. Draw the left knee toward chest until the thigh is parallel to the ground, and simultaneously swing the right arm forward and the left arm backward. Engage your quad to extend the left leg straight out. Return to standing; and then repeat with the opposite leg. 
5. Front lunge: Step the right leg in the front and lower into a lunge, while making sure to keep the knee behind the toes at all times. Push the back through the heel and glutes to a standing position, and then repeat with the opposite side.

6. Lateral lunge: The feet slightly wider than the hip-width apart. Lunge to the right with the right leg, and then return to the starting position, pressing up through the heel and engaging glutes; and then repeat with the opposite side.

PL-WU Protocol. The PL-WU protocol consisted of 5 minutes of jogging followed by double- and single-leg jumps where participants were instructed to maximize jumping height or distance during each exercise. ${ }^{18,27}$ The exercises were adapted to our participants by reducing the difficulty and the intensity. The participant performed each exercise 8 times for each leg, for 3 repetitions with 10-second muscle release between each repetition. The PL exercises were:

1. Bounding exercise: Long jumps using the arms, with little hops in between to get the balance and continue jumping forward.

2. Leg bounding: Run forward with long steps. Balance on one leg and bound forward, landing on the opposite leg. Repeat the bounding motion with control, using the arms and alternating legs.

3. Tuck jumps: Standing with feet hip-distance apart, start by hopping with both the feet off the ground and landing on both feet at the same time.

4. Jump lunges: Start in the low position of a squat with the right foot in front. On the jump, change the leg quickly.

5. Burpees: Lower into a deep squat position. Kick the legs straight out behind and immediately lower the body down, bending at the elbows. Use the arms to quickly push the body and jump straight up, reaching the arms overhead.

6. Lateral bounds: Start in a squatting position, balancing on one leg and then perform an explosive jump as high as possible and far to the left as possible, and then repeat with the opposite leg.

\section{Statistical Analysis}

The statistical analysis of the data is carried out using SPSS software (version 25.0; IBM Corp, Armonk, NY). The intratester reliability of the SEBT composite scores and all reach directions was analyzed using the intraclass correlation coefficient. The normality of the data was verified using the Shapiro-Wilk test. The SEBT scores (the composite and each reach direction) were analyzed using a 2-way analysis of variance (session $\times \mathrm{WU}$ method) with repeated measures. The session factor has 3 levels (pre-WU, post-WU, and 15 min post-WU) and the WU method factor has 2 levels (DS-WU and PL-WU). For each main factor and interaction effect, Bonferroni post hoc test was performed. The effect sizes were calculated as partial eta squared $\eta_{\mathrm{p}}^{2}$ to assess the practical significance of our findings. The alpha level of statistical significance was set at $P<.05$.

\section{Results}

The intratester reliability on the SEBT composite scores and all reach directions was good to excellent ${ }^{28}$ with an intraclass correlation coefficient of .89 to .97 .

In the SEBT composite scores, the 2-way analysis of variance with repeated-measures showed a significant main effect of session $\left(F=42.86, P<.01, \eta_{\mathrm{p}}^{2}=.79\right)$ and WU method $(F=29.69, P<.01$, $\left.\eta_{\mathrm{p}}^{2}=.85\right)$ and a significant (session $\times \mathrm{WU}$ method) interaction $\left(F=72.86, P<.01, \eta_{\mathrm{p}}^{2}=.93\right)$.

Concerning the session effect, the post hoc analysis showed that the SEBT composite scores increased significantly postWU $(P<.05)$ and at 15 minutes post-WU $(P<.05)$ following DS-WU. However, there was no significant difference between the two post-WU sessions (post-WU and $15 \mathrm{~min}$ post-WU). For PL-WU, post hoc analysis showed that the SEBT composite scores decreased significantly post-WU $(P<.05)$, and then increased significantly at 15 minutes post-WU compared with those obtained at the pre-WU session $(P<.05$; Figure 1$)$. Regarding the WU method effect, post hoc analysis showed that the SEBT composite scores were significantly higher $(P<.05)$ in DS-WU than PL-WU at both post-WU sessions.

In SEBT scores (ANT, PLAT, POST, PMED, MED, and AMED), the 2-way analysis of variance with repeated-measures revealed significant main effects of session $(F=13.02, F=14.23$, $\left.F=16.96, F=8.49, F=6.76, F=17.66, P<.05, .57<\eta_{\mathrm{p}}^{2}<.77\right)$ and WU method $(F=7.71, F=14.57, F=21.72, F=6.51, F=11.48$, $\left.F=16.00, P<.05, .37<\eta_{\mathrm{p}}^{2}<.66, P<.05\right)$ as well a significant (session $\times$ WU method) interaction $(F=11.44, F=15.46, F=8.96$, $\left.F=10.67, F=10.71, F=10.38, P<.05, .64<\eta_{\mathrm{p}}^{2}<.75\right)$.

The Bonferroni test showed a significant increase in the SEBT scores in the PLAT, POST, PMED, MED, AMED directions $(P<.05)$ at post-WU and at 15 minutes post-WU $(P<.05)$, but not in ANT, ALAT, and LAT (Table 1). There was no significant difference in all SEBT scores between the 2 post-WU sessions. However, in PL-WU, there was no significant difference in all SEBT scores between pre-WU and post-WU, but at 15 minutes post-WU, these scores increased significantly $(P<.05)$ in all directions (except for ALAT and LAT directions). Concerning the WU method effect (Figure 2), post hoc analysis showed that all SEBT scores (except ALAT and LAT directions) were significantly higher $(P<.05)$ in DS-WU than in PL-WU protocol at the post-WU session. However, at the 15 minutes post-WU, there was no significant difference between these 2 WUs in SEBT scores (except in the POST and PLAT reaching directions; Figure 2).

\section{Discussion}

The purpose of this study was to investigate the effects of DS-WU and PL-WU on dynamic balance in athletes with ID. The major finding was that all SEBT scores (except ANT, ALAT, and LAT directions) improved immediately and at 15 minutes after the DSWU. However, these scores did not change immediately after PLWU, but increased 15 minutes later. Interestingly, DS-WU induced a better improvement in SEBT scores than PL-WU (in composite scores and in all reaching directions) immediately after the WU session. This difference was maintained for 15 minutes after the WU session only for the SEBT composite scores.

DS-WU resulted in a significant improvement in all SEBT scores (except ANT, ALAT, and LAT directions), immediately as well as 15 minutes after the WU session. This result is in accordance with previous studies that investigated the effect of DS on balance performance. In this context, Nejati et $\mathrm{al}^{29}$ reported that DS-WU, compared with nonstretching exercises, induces a better static and dynamic balance performance and agility in athletes with typical development. Similarly, a positive effect of DS on dynamic balance (SEBT scores), compared with nonstretching exercises, has been found in physical education and sports students. ${ }^{30}$ To the best of our knowledge, there is only one study on DS in adults with ID. ${ }^{31}$ This study investigated the effect of DS exercises on 


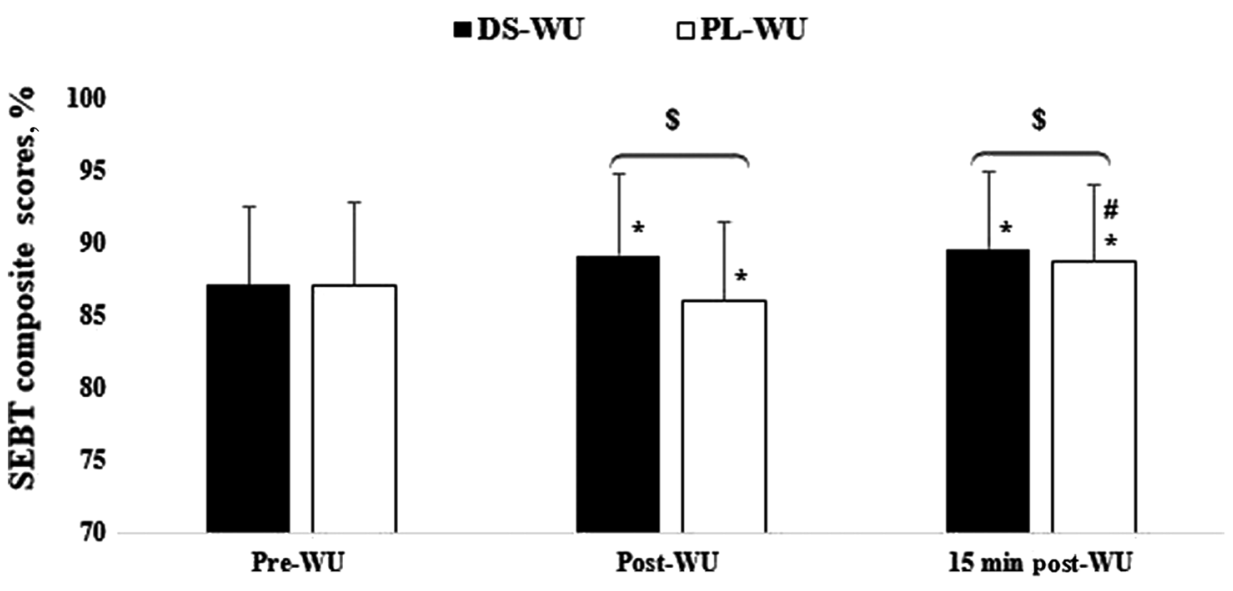

Figure 1 - The SEBT composite scores at pre-WU, post-WU, and 15 minutes post-WU between DS-WU and PL-WU. DS indicates dynamic stretching; PL, plyometric; SEBT, Star Excursion Balance Test; WU, warm-up. *Statistical difference between pre-WU and the other sessions (post-WU and 15 min post-WU) $(P<.05)$. ${ }^{\#}$ Statistical difference between post-WU and 15 minutes post-WU sessions $(P<.05)$. ${ }^{\$}$ Statistical difference between DS-WU and PL-WU $(P<.05)$.

Table 1 Scores Values of SEBT of 2 Different WU Methods at Pre-WU, Post-WU, and at 15 Minutes Post-WU Sessions

\begin{tabular}{|c|c|c|c|c|}
\hline \multirow[b]{2}{*}{ WU method } & \multirow[b]{2}{*}{ Directions } & \multicolumn{3}{|c|}{$\%$ Normalized reach distances, mean (SD) } \\
\hline & & Pre-WU & Post-WU & $15 \mathrm{~min}$ post-WU \\
\hline \multirow[t]{8}{*}{ DS-WU } & ANT & $82.10(6.79)$ & $84.02(6.83)$ & $84.42(6.35)$ \\
\hline & ALAT & $65.86(8.31)$ & $66.97(8.90)$ & $66.92(8.63)$ \\
\hline & LAT & $70.29(8.50)$ & $70.73(8.40$ & $71.14(7.69)$ \\
\hline & PLAT & $91.14(8.27)$ & $94.39(7.18)^{*}$ & $95.22(7.76)^{*}$ \\
\hline & POST & $99.01(8.20)$ & $102.16(7.78)^{*}$ & $102.83(7.53)^{*}$ \\
\hline & PMED & $99.42(7.46)$ & $101.68(7.16)^{*}$ & $101.87(7.42)^{*}$ \\
\hline & MED & $100.68(7.44)$ & $102.89(7.50)^{*}$ & $103.35(7.06)^{*}$ \\
\hline & AMED & $87.83(7.24)$ & $90.12(7.28)^{*}$ & $90.61(7.85)^{*}$ \\
\hline \multirow[t]{8}{*}{ PL-WU } & ANT & $81.74(6.73)$ & 81.09 (5.66) & $83.89(5.69) * * *$ \\
\hline & ALAT & $66.17(7.56)$ & $65.94(7.28)$ & $66.76(6.89)$ \\
\hline & LAT & $70.60(8.15)$ & $69.54(9.61)$ & $70.70(8.61)$ \\
\hline & PLAT & $91.03(8.63)$ & $90.61(9.10)$ & $93.30(8.27)^{* * * *}$ \\
\hline & POST & $98.60(7.85)$ & $98.07(8.42)$ & $100.75(7.59)^{* * * *}$ \\
\hline & PMED & $99.60(7.79)$ & $98.33(7.14)$ & $100.61(7.90)^{* * * *}$ \\
\hline & MED & $100.31(6.66)$ & $99.52(6.64)$ & $101.86(6.73)^{* * * *}$ \\
\hline & AMED & $87.92(7.13)$ & $86.84(6.73)$ & $89.44(6.86)^{* * * *}$ \\
\hline
\end{tabular}

Abbreviations: ALAT, anterolateral; AMED, anteromedial; ANT, anterior; DS, dynamic stretching; LAT, lateral; MED, medial; PL, plyometric; PLAT, posterolateral; PMED, posteromedial; POST, posterior; SEBT, Star Excursion Balance Test; WU, warm-up.

*Statistical difference between pre-WU and other sessions (post-WU and 15 min post-WU) $(P<.05)$. **Statistical difference between post-WU and 15 minutes post-WU sessions $(P<.05)$.

flexibility and balance. The result showed that DS exercises were effective in improving flexibility and postural balance of all the participants at the posttest measurements compared with the pretest measurements. A number of mechanisms have been proposed to explain the effect of DS-WU on balance performance. Indeed, it has been reported that DS increases the muscle temperature, ${ }^{32}$ as well as the nerve receptors sensitivity and the nerve conduction velocity, ${ }^{17}$ resulting in faster and more powerful muscle contraction in the SEBT. ${ }^{17}$ It has been proposed that an increase in the temperature may decrease the viscous resistance of muscle and consequently enhance the tissue extensibility that is associated with better SEBT scores. ${ }^{33} \mathrm{DS}$ might also produce postactivation potentiation; that is, a transient improvement of muscular contractility following a conditioning voluntary contraction. ${ }^{34}$ The main mechanism of postactivation potentiation is a higher rate of cross bridging. ${ }^{35,36}$ Such a rate relies on the phosphorylation of myosin regulatory light chains that renders actin-myosin interaction more sensitive to $\mathrm{Ca}^{2+}$ release from the sarcoplasmic reticulum. ${ }^{34}$ The postactivation potentiation may reduce the time to peak torque and increase the rate of torque development, increasing muscular force, power, and speed in subsequent performance, ${ }^{34}$ and facilitating the motor neuron activity. ${ }^{17}$ All these factors may improve dynamic balance performance. ${ }^{17}$ It 


\section{$\square$ Pre-WU $\square$ Post-WU $\square 15 \mathrm{~min}$ post-WU}

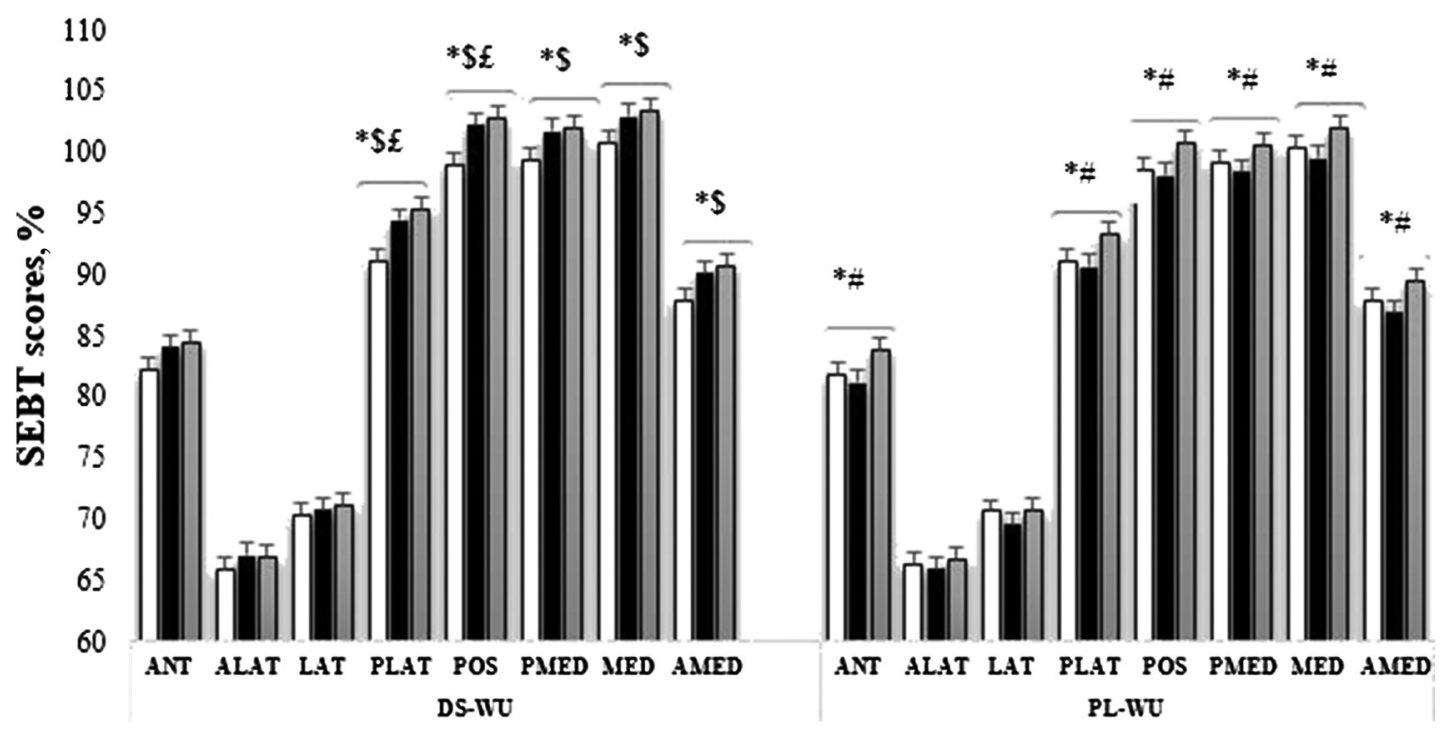

Figure 2 - The SEBT scores of all directions (ALAT, ANT, AMED, MED, PMED, POST, PLAT, and LAT) at pre-WU, post-WU, and 15 minutes post-WU sessions between dynamic stretching warm-up (DS-WU) and plyometric warm-up (PL-WU). ALAT, anterolateral; AMED, anteromedial; ANT, anterior; DS, dynamic stretching; LAT, lateral; MED, medial; PL, plyometric; PLAT, posterolateral; PMED, posteromedial; POST, posterior; SEBT, Star Excursion Balance Test; WU, warm-up. ${ }^{*}$ Statistical difference between pre-WU and the other sessions (post-WU and 15 min post-WU) $(P<.05)$. ${ }^{\#}$ Statistical difference between post-WU and 15 minutes post-WU sessions $(P<.05)$. ${ }^{\$}$ Statistical difference between DS-WU and PL-WU at post-WU $(P<.05) .{ }^{£}$ Statistical difference between DS-WU and PL-WU at 15 minutes post-WU $(P<.05)$.

has been suggested that good balance ability has been significantly associated with reduced risk of injuries. ${ }^{37}$ Furthermore, the SEBT has been considered as a validated tool to predict lower-extremity injuries. ${ }^{11}$ Therefore, SEBT improvements observed in our study, following DS-WU, indicated that this WU is beneficial for sports injuries prevention.

By contrast, PL-WU did not improve the SEBT scores immediately after the WU session. Similarly, in athletes with typical development, Kim et al ${ }^{18}$ indicated that WU exercises such as PL did not improve dynamic balance. According to these authors, fatigue after PL exercises may contribute to this inefficiency. The ability to balance decreases as muscle fatigue accumulates and proprioceptive responses to the joints are inhibited. ${ }^{38} \mathrm{~A}$ previous study showed that unipedal fatigue following a high-intensity exercise alters unipedal postural balance in adults with ID. ${ }^{39}$ It has also been reported that individuals with ID showed greater decline in force immediately after a fatiguing exercise. ${ }^{40}$ It is important to note that the reduced postural balance has been related to an elevated risk of lower limb injury. ${ }^{9}$ Hence, one could argue that the decreased SEBT scores observed immediately after our PLWU could be explained by the fatigue effect, ${ }^{18}$ which may lead to a higher risk of injury and lessened performance. Importantly, 15 minutes following PL-WU, improvement in SEBT scores began to appear (except in the ALAT and LAT directions). Most of the previous studies investigating the effects of 6 to 7 weeks of PL exercises on balance in athletes with typical development reported positive effects of these exercises on dynamic balance. ${ }^{41,42}$ Specifically, when combined with balance/strength exercises, PL training induces an improvement in the lower limb neuromuscular control $^{42}$ as well as in dynamic balance. ${ }^{43}$ Several factors could explain the effect of PL-WU on dynamic balance. The rapid changes in length and tension placed on the tendino-muscular structures during eccentric loading may have facilitated tailoring of the muscle spindles and the Golgi tendon organs. The desensitization of the Golgi tendon organs leads to increased sensitivity of muscle spindles. ${ }^{17}$ Enhancing the sensitivity of the muscle spindle system may increase its afferent contributions to the central nervous system with regard to the joint position. These factors may enhance the SEBT performance. Moreover, in PL exercises, there is a kind of coordination that can improve the balance in achieving the SEBT. ${ }^{17}$

Interestingly, this study indicated that neither DS-WU nor PLWU induced dynamic balance improvement in the challenging directions (ANT, ALAT, and LAT). Indeed, it has been specified that balance depends on the difficulty of the task performed. ${ }^{44}$ It seems that the low neuronal and muscular strength in athletes with ID, compared with their counterparts without ID, ${ }^{45}$ failed to improve significantly the SEBT scores in the challenging directions (ANT, ALAT, and LAT). According to Mohammadi et al, ${ }^{46}$ performing the SEBT in these directions requires good neuromuscular strength.

Specifically, DS-WU and PL-WU affected dynamic balance differently. Actually, 15 minutes after DS-WU or PL-WU, dynamic balance shows similar improvement. However, immediately after the WU session, DS-WU improves dynamic balance performance while PL-WU alters this performance (SEBT composite score). It seems that the balance ability decreased immediately after PL-WU because of fatigue but was rapidly recovered and increased thereafter. ${ }^{18}$

\section{Practical Implications}

The results of this study are likely to have important practical implications. DS-WU and PL-WU could be important methods for the WU routine in athletes with ID as they are both effective to improve dynamic balance. Such an improvement may hopefully 
reduce the incidence of subsequent sport injuries as well as optimize sport performance. In this context, it has been reported that having a good balance not only reduces the risk of falls and sport injuries, but also allows to optimize the performance in several athletic disciplines. ${ }^{37}$ Therefore, coaches for athletes with ID are recommended to incorporate DS-WU and/or PLWU in the WU routine. However, when integrating PL exercises in the WU routine, caution should be used immediately after these exercises as dynamic balance is decreased at this period and increases only after 15 minutes. In this sense, challenging exercises that need good balance should be avoided during the 15 minutes after PL-WU to reduce the risk of injuries.

\section{Limitations of the Study}

This study faces some limitations that should be addressed. Because of recruitment difficulties, only 12 athletes with ID participated in this study. Our sample size should be increased in future studies to obtain generative results. Our 2 WU methods included 5 minutes of jogging. A possible contribution of this jogging to improvement of dynamic balance following the WU exercises could be advanced. A testing session post jogging could be considered in future studies to control the possible effect of jogging on dynamic balance. Moreover, risk of injuries surveillance before and/or during a competition period is important because there is a need to document safe participation of these athletes in competition. It has been reported that $3.5 \%$ of athletes participating in the Special Olympics games received injuries/illness care during the games. ${ }^{4}$ For this purpose, understanding the effects of DS-WU and PL-WU on balance performance and injury incidence during a training period (precompetitive) would be of interest to prevent sport injuries that could occur during training and/or competition.

\section{Conclusion}

Overall, this study demonstrated that both DS-WU and PL-WU could improve dynamic balance performance in athletes with ID. These improvements were not observed in the challenging direction (ALAT and LAT) for DS-WU or PL-WU. Importantly, immediately after the WU session, DS-WU induces dynamic balance improvement, whereas PL-WU alters balance performance. However, 15 minutes after performing the $2 \mathrm{WU}$ methods, dynamic balance shows a similar improvement. Future studies investigating these effects for a prolonged period are needed. Analysis of these WUs in injury incidence will also be useful.

\section{Acknowledgments}

The authors would like to thank all participants for their understanding and availability. They thank all collaborators and volunteers of the "Association of Tunisian Parents and Mentally Handicapped Friends" for their contribution in this study. No potential conflict of interest was reported by the authors. This research did not receive any specific grant from funding agencies in the public, commercial, or not-for-profit sectors.

\section{References}

1. Surjus LTdL, Campos RTO. Interface entre deficiência intelectual e saúde mental: revisão hermenêutica. Rev Saúde Públ. 2014;48(3):532540. PubMed ID: 32429428 doi:10.1590/S0034-8910.2014048004711
2. Pestana MB, Barbieri FA, Vitório R, Figueiredo GA, MauerbergdeCastro E. Effects of physical exercise for adults with intellectual disabilities: a systematic review. J Phys Educ. 2018;41(29):e2920.

3. Fagher K, Lexell J. Sports-related injuries in athletes with disabilities. Scand J Med Sci Sports. 2014;24(5):e320-e331. PubMed ID: 24422719 doi:10.1111/sms.12175

4. Ng KL. Perception of Special Olympics coaches on safety in their training of their Special Olympics athletes [dissertation]. Prague: Charles University; 2009.

5. Dellavia C, Pallavera A, Orlando F, Sforza C. Postural stability of athletes in Special Olympics. Percept Motor Skills. 2009;108(2): 608-622. PubMed ID: 19544966 doi:10.2466/pms.108.2.608-622

6. Little J-A, Woodhouse JM, Lauritzen JS, Saunders KJ. The impact of optical factors on resolution acuity in children with Down syndrome. Invest Ophthalmol Vis Sci. 2007;48(9):3995-4001. PubMed ID: 17724178 doi:10.1167/iovs.06-1387

7. Hale L, Miller R, Barach A, Skinner M, Gray A. Motor control test responses to balance perturbations in adults with an intellectual disability. J Intellect Dev Disabil. 2009;34(1):81-86. PubMed ID: 19234981 doi: $10.1080 / 13668250802683810$

8. Zur O, Ronen A, Melzer I, Carmeli E. Vestibulo-ocular response and balance control in children and young adults with mild-to-moderate intellectual and developmental disability: a pilot study. Res Dev Disabil. 2013;34(6):1951-1957. PubMed ID: 23584174 doi:10. 1016/j.ridd.2013.03.007

9. Hrysomallis C. Relationship between balance ability, training and sports injury risk. Sports Med. 2007;37(6):547-556. PubMed ID: 17503879 doi:10.2165/00007256-200737060-00007

10. Butler RJ, Southers C, Gorman PP, Kiesel KB, Plisky PJ. Differences in soccer players' dynamic balance across levels of competition. J Athl Train. 2012;47(6):616-620. PubMed ID: 23182008 doi:10. 4085/1062-6050-47.5.14

11. Gribble PA, Hertel J, Plisky P. Using the star excursion balance test to assess dynamic postural-control deficits and outcomes in lower extremity injury: a literature and systematic review. J Athl Train. 2012;47(3):339-357. PubMed ID: 22892416 doi:10.4085/10626050-47.3.08

12. Winchester JB, Nelson AG, Landin D, Young MA, Schexnayder IC. Static stretching impairs sprint performance in collegiate track and field athletes. J Strength Cond Res. 2008;22(1):13-19. PubMed ID: 18296950 doi:10.1519/JSC.0b013e31815ef202

13. McMillian DJ, Moore JH, Hatler BS, Taylor DC. Dynamic vs. staticstretching warm up: the effect on power and agility performance. $J$ Strength Cond Res. 2006;20(3):492-499. PubMed ID: 16937960

14. Costa PB, Graves BS, Whitehurst M, Jacobs PL. The acute effects of different durations of static stretching on dynamic balance performance. J Strength Cond Res. 2009;23(1):141-147. PubMed ID: 19077736 doi:10.1519/JSC.0b013e31818eb052

15. Behm DG, Chaouachi A. A review of the acute effects of static and dynamic stretching on performance. Eur J Appl Physiol. 2011;111(11):26332651. PubMed ID: 21373870 doi:10.1007/s00421-011-1879-2

16. Perry M. Try This Full-Body Dynamic Warm-Up to Prep for Any Workout. http://greatist.com/fitness/full-body-dynamic-warmup. Accessed from August, 2013.

17. Razeghi M, Hamoule E. Comparison of the immediate effects of modified and routine warm-ups on knee joint function and dynamic balance in athletes. J Rehabil Sci Res. 2017;3(4):97-102.

18. Kim K, Lee T, Kang G, Kwon S, Choi S, Park S. The effects of diverse warm-up exercises on balance. J Phys Ther Sci. 2014;26(10):16011603. PubMed ID: 25364123 doi:10.1589/jpts.26.1601

19. Fatouros IG, Jamurtas AZ, Leontsini D, et al. Evaluation of plyometric exercise training, weight training, and their combination on vertical 
jumping performance and leg strength. J Strength Cond Res. 2000; 14(4):470-476.

20. Kotzamanidis C. Effect of plyometric training on running performance and vertical jumping in prepubertal boys. $J$ Strength Cond Res. 2006;20(2):441-445. PubMed ID: 16686577

21. Witzke KA, Snow CM. Effects of plyometric jump training on bone mass in adolescent girls. Med Sci Sports Exerc. 2000;32(6):1051-1057. PubMed ID: 10862529 doi:10.1097/00005768-200006000-00003

22. Chu DA. Jumping Into Plyometrics. Champaign, IL: Human Kinetics; 1998.

23. Wechsler D. Wechsler Adult Intelligence Scale. San Antonio, TX: NCS Pearson, Inc; 2008.

24. Kinzey SJ, Armstrong CW. The reliability of the star-excursion test in assessing dynamic balance. J Orthop Sports Phys Ther. 1998;27(5):356-360. PubMed ID: 9580895 doi:10.2519/jospt. 1998.27.5.356

25. Faigenbaum AD, McFarland JE, Schwerdtman JA, Ratamess NA, Kang J, Hoffman JR. Dynamic warm-up protocols, with and without a weighted vest, and fitness performance in high school female athletes. J Athl Train. 2006;41(4):357. PubMed ID: 17273458.

26. Mann DP, Jones MT. Guidelines to the implementation of a dynamic stretching program. Strength Cond J. 1999;21(6):53-58. doi:10. 1519/00126548-199912000-00014

27. Masamoto N, Larson R, Gates T, Faigenbaum A. Acute effects of plyometric exercise on maximum squat performance in male athletes. $J$ Strength Cond Res. 2003;17(1):68-71. PubMed ID: 12580658

28. Hyong IH, Kim JH. Test of intrarater and interrater reliability for the star excursion balance test. J Phys Ther Sci. 2014;26(8):1139-1141. PubMed ID: 25202168 doi:10.1589/jpts.26.1139

29. Nejati N, Balarin GB, Gonzolas A, Neal J. Acute effects of dynamic and static stretching during warm-up on balance and agility for untrained adults. In: 11th Annual Symposium on Graduate Research and Scholarly Projects, Wichita State University; 2015.

30. Erkut O, Gelen E, Sunar C. Acute effects of different warm-up methods on dynamic balance. Int J Sports Sci. 2017;7(3):99-104.

31. Kurt Ö, Çuhadar S. Effects of most to least prompting procedure on teaching exercise for adults with intellectual disabilities. J Educ Train Stud. 2018;6(9a):1-14. doi:10.11114/jets.v6i9a.3485

32. Bishop D. Warm-up II: Performance changes following active warm up on exercise performance. Sports Med. 2003;33(1):483-498. doi:10. 2165/00007256-200333070-00002

33. Opplert J, Babault N. Acute effects of dynamic stretching on muscle flexibility and performance: an analysis of the current literature. Sports Med. 2018;48(2):299-325. PubMed ID: 29063454 doi:10. 1007/s40279-017-0797-9

34. Sale DG. Postactivation potentiation: role in human performance. Exerc Sport Sci Rev. 2002;30(3):138-143. PubMed ID: 12150573 doi:10.1097/00003677-200207000-00008
35. Baudry S, Duchateau J. Postactivation potentiation in a human muscle: effect on the rate of torque development of tetanic and voluntary isometric contractions. J Appl Physiol. 2007;102(4): 1394-1401. PubMed ID: 17204572 doi:10.1152/japplphysiol. 01254.2006

36. Cè E, Rampichini S, Maggioni MA, Veicsteinas A, Merati G. Effects of passive stretching on post-activation potentiation and fibre conduction velocity of biceps brachii muscle. Sport Sci Health. 2008; 4(3):43-50. doi:10.1007/s11332-008-0066-2

37. Hrysomallis C. Balance ability and athletic performance. Sports Med. 2011;41(3):221-232. PubMed ID: 21395364 doi:10.2165/11538560000000000-00000

38. Inoue $\mathrm{K}$, Uematsu M, Maruoka $\mathrm{H}$, et al. Influence of lower limb muscle fatigue on balance function. J Phys Ther Sci. 2013;25(3): 331-335. doi:10.1589/jpts.25.331

39. Borji R, Rebai H, Baccouch R, Laatar R, Sahli S. Unilateral fatigue affects the unipedal postural balance in individuals with intellectual disability. J Motor Behav. 2017;49(4):407-413. doi:10.1080/ 00222895.2016.1219309

40. Borji R, Sahli S, Zarrouk N, Zghal F, Rebai H. Neuromuscular fatigue during high-intensity intermittent exercise in individuals with intellectual disability. Res Dev Disabil. 2013;34(12):4477-4484. PubMed ID: 24139713 doi:10.1016/j.ridd.2013.09.025

41. Asadi A, de Villarreal ES, Arazi H. The effects of plyometric type neuromuscular training on postural control performance of male team basketball players. J Strength Cond Res. 2015;29(7): 1870-1875. PubMed ID: 25563677 doi:10.1519/JSC.0000000000 000832

42. Myer GD, Ford KR, Brent JL, Hewett TE. The effects of plyometric vs dynamic stabilization and balance training on power, balance, and landing force in female athletes. J Strength Cond Res. 2006;20(2):345. PubMed ID: 16686562

43. Hadi H, Farhady H, Bashiri M. Effect of six-week strength and plyometric training on dynamic balance of male athletic students. $J$ Rehabil Sci Res. 2012;7(2):215-224.

44. Streepey JW, Angulo-Kinzler RM. The role of task difficulty in the control of dynamic balance in children and adults. Hum Mov Sci. 2002;21(4):423-438. PubMed ID: 12450677 doi:10.1016/S01679457(02)00104-5

45. Borji R, Zghal F, Zarrouk N, Sahli S, Rebai H. Individuals with intellectual disability have lower voluntary muscle activation level. Res Dev Disabil. 2014;35(12):3574-3581. PubMed ID: 25241117 doi:10.1016/j.ridd.2014.08.038

46. Mohammadi V, Hilfiker R, Jafarnezhadgero AA, Jamialahmadi S, Karimizadeh Ardakani M, Granacher U. Relationship between training-induced changes in the star excursion balance test and the Y balance test in young male athletes. Ann Appl Sport Sci. 2017;5(3):31-38. doi:10.29252/acadpub.aassjournal.5.3.31 